\title{
Ética militar y geopolítica. Una mirada a la ética militar en las políticas institucionales ${ }^{1}$
}

\author{
https://doi.org/10.21830/9789585377134.01
}

\author{
Jesús Eduardo Moreno Peláez \\ Ximena Andrea Cujabante Villamiß \\ Humberto Librado Castillo 4 \\ Escuela Superior de Guerra "General Rafael Reyes Prieto"
}

\section{Resumen}

El presente capítulo de libro aborda la relación entre la ética militar y la geopolítica, con especial énfasis en el caso colombiano. Para el desarrollo de esta investigación se utilizó una metodología cualitativa deductiva. En este sentido, en la primera parte se aborda una dimensión general sobre la comprensión de la ética militar y la geopolítica. En la segunda se examinan los aspectos relacionados con la ética militar en el desarrollo institucional colombiano desde los marcos constitucionales, así como las políticas derivadas de dicho marco, hasta los documentos centrales del Ejército en su componente estratégico. Finalmente, la tercera parte muestra de qué forma esta dimensión se relaciona con los principales retos geopolíticos del país. Entre las conclusiones a las que se llegó vale

1 Este capítulo presenta los resultados colaborativos de dos proyectos de investigación: (1) "Ética militar en entornos complejos de seguridad y defensa: lecturas y aportes desde la experiencia de las Fuerzas Militares de Colombia”, del grupo de investigación Masa Crítica de la Escuela Superior de Guerra "General Rafael Reyes Prieto", Colombia, categorizado en B por Minciencias y con código de registro COL0123247, y (2) "Mujeres de arma, seguridad y defensa nacional. Un análisis desde sus percepciones", del grupo de investigación en Ciencias Militares de la Escuela Militar de Cadetes "General José María Córdova”, Colombia, categorizado en B por Minciencias y con código de registro COL0082556. Los puntos de vista pertenecen a los autores y no reflejan necesariamente los de las instituciones participantes.

2 Teniente Coronel (RA) del Ejército Nacional de Colombia. Magíster en Inteligencia Estratégica de la Escuela de Inteligencia y Contrainteligencia "Bg. Ricardo Charry Solano". Magíster en Seguridad y Defensa Nacionales de la Escuela Superior de Guerra "General Rafael Reyes Prieto". Profesional en Ciencias Militares de la Escuela Militar de Cadetes "General José María Córdova”. OrciD: https://orcid. org/0000-0001-8516-3893 - Contacto: jesus.moreno@esdegue.edu.co

$3 \mathrm{PhD}$ en Estudios Políticos y magíster en Asuntos Internacionales de la Universidad Externado de Colombia. Especialista en Negociación y Relaciones Internacionales de la Universidad de los Andes. Politóloga de la Universidad Javeriana. OrCID: https://orcid.org/0000-0002-5473-163X - Contacto: ximena. cujabante@unimilitar.edu.co

$4 \mathrm{PhD}$ (c) en Estudios Políticos y magíster en Análisis de Problemas Políticos, Económicos e Internacionales de la Universidad Externado de Colombia. Politólogo de la Universidad Nacional de Colombia. OrciD: https://orcid.org/0000-0001-8431-8611 - Contacto: humberto.librado@esdegue.edu.co 
la pena mencionar que la reflexión sobre la consolidación y la proyección geopolítica de un Estado tiene un papel muy importante en el control y la administración de la violencia en el territorio, lo cual hace que las Fuerzas Armadas sean un actor fundamental en este tipo de procesos.

Palabras clave: estrategia; ética militar; geopolítica; poder.

\section{Introducción}

Desde la perspectiva de la geopolítica, son muchos los aspectos que se priorizan en el campo estratégico de los Estados. Así, se consideran centrales los recursos naturales, el aprovechamiento de situaciones geográficas estratégicas y el desarrollo de acciones que, en un caso como el de Colombia, permiten la consolidación territorial del Estado. En este escenario, el personal militar es un actor fundamental, pues contribuye al despliegue y control del territorio que busca garantizar la prosperidad del país y el aprovechamiento de su potencial geopolítico.

En este sentido, resulta crucial aproximarse a la manera en que su labor también se relaciona con una serie de decisiones que implican una profunda reflexión de la actuación vinculada con la dimensión del ejercicio de la violencia de los Estados. Desde este reconocimiento, el presente capítulo analiza cómo en este necesario despliegue de las Fuerzas Armadas en el territorio los individuos e instituciones intervinientes no pueden dejar de lado la ética.

Específicamente, el capítulo estudia la forma en que la dimensión ética militar se presenta en los principios de las Fuerzas Armadas y se evidencia en los lineamientos generales de las políticas institucionales, lo cual muestra que la relación entre geopolítica y ética militar no se subsume en una visión retórica del quehacer de las organizaciones relacionadas, sino que, aún más allá, la ética se constituye en premisa del despliegue que permite la consolidación geopolítica.

Con este fin, el texto se divide en tres secciones: la primera analiza algunas nociones generales sobre ética militar y geopolítica; posteriormente, la segunda sección indaga la ética militar en las políticas institucionales y, finalmente, la tercera estudia las implicaciones de esta dimensión en la geopolítica del Estado en el marco de algunos de sus principales retos. 


\section{Ética militar}

La ética es un saber que tiene como fundamento orientar la acción humana hacia lo bueno, lo correcto. Es así como en el campo militar la ética desempeña un papel primordial que conecta los aspectos técnicos, jurídicos y sociales. Entonces, la ética militar se refiere a los

cimientos morales de la profesión militar, los valores que constituyen su núcleo, los principios que guían a los que ejercen esa profesión y las responsabilidades esenciales que deben asumir en la defensa de su país. Se refiere a lo que hacen y cómo lo desarrollan, pero también a lo que espera de su comportamiento, a sus relaciones profesionales y a la percepción que tienen de sus conciudadanos. (Moliner, 2020, p. 77)

En este orden de ideas, la teoría de la guerra justa es un claro ejemplo de la ética militar como cuerpo doctrinal. Los principios del ius ad bellum y del ius in bello se han constituido en un referente internacional cuando de regular la guerra se trata. De esta forma, la necesidad de la ética militar se fundamenta en que permite llegar, mediante la razón y el juicio moral, al conocimiento de que el ejercicio de la violencia en el combate debe estar justificado en razones éticas. Asimismo, se pone de manifiesto en la atención que los miembros de las Fuerzas Armadas deben prestar a los cambios en los principios y valores sociales y culturales, tanto de su sociedad como del resto de países (Moliner, 2018a, p. 31).

La teoría de la guerra justa consta de juicios que tienen que ver con qué tan justo es ir a la guerra (ius ad bellum) y la justicia en la conducción de la guerra (ius in bello). En este sentido, la teoría se basa en el uso sistemático del razonamiento moral para tomar una decisión de emprender un conflicto armado con otro Estado, incluyendo el accionar durante y después de la guerra (Wead, 2015). Es así como los principios de la guerra justa ofrecen los motivos éticos, morales, psicológicos, prácticos y estratégicos para la conducción de la guerra yuxtapuestos con los motivos y las acciones de los enemigos. Esta codificación de la guerra justa tiene lugar a través de tratados y compromisos internacionales pertinentes a diferentes situaciones de batalla (Wead, 2015).

Hoy por hoy, el sistema internacional se caracteriza por avances tecnológicos sin precedentes que permiten la interconexión permanente entre los 
diferentes actores del sistema internacional, así como avances en las capacidades militares de los Estados. Además, dado que la paz es un objetivo más que estatal, la ética militar cada vez cobra mayor relevancia ante la necesidad de dotar a los militares de capacidades morales y de conocimientos que se ajusten a los requisitos de la profesión en el marco del entorno mundial y los cambios tecnológicos.

Es así como en el sistema internacional contemporáneo la ética militar debe afrontar algunos riesgos: (1) la integración en códigos, estatutos de valores y principios que faciliten conductas en el marco de la ética. (2) La necesidad de contar con la metodología adecuada en la formación de los profesionales de las armas, de tal manera que les permita adquirir una capacitación ética que sirva de base para la toma de decisiones en el ejercicio de sus funciones. (3) Todos aquellos asuntos relacionados con el ius ad bellum, como la relevancia del principio de causa justa y, por extensión, de guerra justa con los nuevos desarrollos que presentan los conflictos bélicos. (4) Todos aquellos asuntos enmarcados en el ius in bello, como por ejemplo los problemas éticos relacionados con el avance de la ciencia y la tecnología, que no solo afectan el desarrollo de la guerra y la forma de llevarla a cabo, sino también el origen de los conflictos. (5) Aquellos asuntos respecto al ius post bellum, como la necesidad de tener en cuenta el objetivo ético de avanzar hacia la paz (Moliner, 2018a).

Dentro de las características de la ética militar se encuentran (Moliner, 2018a):

- Se trata de una ética profesional sustentada en principios y valores. Su objetivo es ayudar a los militares en el desarrollo de sus funciones y tareas ante desafíos morales a los que tienen que enfrentarse en el ejercicio de su profesión.

- La ética militar proporciona una motivación y una convicción moral para mantener los valores y las actitudes que exige la milicia.

- La ética militar le facilita al profesional unas capacidades y conocimientos necesarios para evaluar, desde planteamientos morales, los límites de su conducta profesional y orientarla teniendo en cuenta la guerra justa, las normas morales a tener en cuenta en los conflictos 
armados y la adecuación ética de la evolución técnica y táctica de las capacidades militares (Moliner, 2018a).

- Otro de los propósitos de la ética militar es fundar e inspirar conductas y actitudes que forman parte de la moral de victoria.

- La ética militar tiene un carácter multidisciplinar.

- La perspectiva metodológica que emplea la ética militar debe ser teórica, científica, empírica y reflexiva.

\section{Geopolítica}

Del mismo modo en que se analizó previamente la dimensión de la ética militar desde una visión amplia que permita operacionalizar su uso para efectos del presente trabajo, a continuación se aborda la geopolítica.

La geopolítica es una disciplina que busca comprender la relación compleja entre el espacio, el territorio y las múltiples decisiones de carácter político. Estas decisiones políticas tienen como actor natural a los Estados modernos, dada su preeminencia frente a otro tipo de actores, especialmente en el espacio de los países. No obstante, también se tienen en cuenta otro tipo de actores políticos que tienen la capacidad de generar efectos en la transformación territorial.

Es importante entender que la geopolítica aborda fundamentalmente tres tipos de escala para construir sus análisis: la primera se refiere a la escala global, relacionada con la comprensión de problemas y fenómenos que tienen lugar entre los Estados. La segunda escala hace referencia a lo estatal, que corresponde al análisis de las problemáticas internas a un Estado. Finalmente, la escala local o regional reconoce aspectos mucho más específicos, tales como los departamentos o los municipios. Estas múltiples escalas y sus dinámicas se relacionan entre sí y se afectan mutuamente (Lacoste, 2009; citado por Núnez \& Ferro, 2017).

La geopolítica representa un área de trabajo de carácter interdisciplinar que recoge los conocimientos de la ciencia política, la geografía, la sociología, la inteligencia estratégica y diferentes dimensiones de la geografía, como la geografía económica y la geografía urbana. Este tipo de análisis de carácter 
interdisciplinario le ha permitido a la geopolítica tener una presencia activa en los procesos de toma de decisión de los gobiernos y vincularse a diferentes áreas de análisis para la actuación de los gobiernos en las escalas precitadas.

En este sentido, la geopolítica permite una riqueza de análisis en factores centrales que se relacionan con la estabilidad de los Estados. De este modo, contemporáneamente asume un papel central para el estudio de aspectos como la seguridad y la soberanía nacional, las relaciones internacionales, los conflictos interestatales y las inversiones globales (Núñez \& Ferro, 2017).

Siguiendo a Ballesteros et al. (2014), la geopolítica "sirve para analizar el escenario en que debe desarrollar su campo de acción. Su objetivo es facilitar la toma de las decisiones políticas, proporcionando el conjunto de informaciones que faciliten el trabajo de los decisores" (p. 3). Estas informaciones permiten un conocimiento mayor de la territorialidad en cuanto espacio para el desarrollo de las relaciones sociales y las actividades humanas en un país, así como posibilitan la comprensión de las relaciones de poder en su carácter territorializado.

Desde otras aproximaciones, también se puede entender la geopolítica como una disciplina que se encarga de analizar la forma en que los actores dentro de un sistema determinado interactúan con el fin de obtener poder y de esta forma influir a los demás (Baños, 2016). En este sentido, se puede afirmar que el centro de la geopolítica consiste en determinar cuáles son esas fuentes de poder y cómo las usan los Estados, junto con los demás actores dentro del sistema internacional.

Debido al dinamismo de la geopolítica, esta disciplina se aborda desde distintas miradas, ya que se ha transformado y adaptado progresivamente a las diversas circunstancias y visiones de quienes la estudian, de manera que ha avanzado acorde a los cambios políticos de la sociedad y su entorno geográfico. Al respecto, John Agnew (2005; citado por Aragón, 2010) plantea que la geopolítica es el "estudio de la repercusión de las distribuciones y divisiones geográficas en la marcha de la política mundial” (p. 5).

De otra parte, Leiva (2014) considera que

la geopolítica es una ciencia de suma utilidad para la correcta información de las personas; es insustituible para los elementos militares que la utilizan como 
marco general para sus apreciaciones estratégicas; es indispensable para todos quienes intervienen en las orientaciones de la política exterior de los Estados y absolutamente necesaria para adoptar modelos de desarrollo. (p. 1)

Este autor también presenta otro concepto acuñado de geopolítica:

Ciencia que pretende fundar la política nacional o internacional en el estudio sistemático de los factores geográficos, económicos, raciales, culturales y religiosos; la geopolítica es la relación que existe entre el poder y su distribución en diferentes zonas de influencia, donde con anterioridad las dominaciones coloniales, las guerras y el uso de la fuerza determinaban estas zonas de influencia. (Leiva, 2014, p. 1)

De otra parte, Friedrich Ratzel, geógrafo alemán fundador de la geografía humana o antropogeografía, sostuvo que la geopolítica es la ciencia que establece que las características y las condiciones geográficas, principalmente los grandes espacios, desempeñan un papel decisivo en la vida de los Estados y que el individuo y la sociedad humana dependen del suelo en el que viven, de manera que su destino está determinado por las leyes de la geografía (Grajales, 2010). Asimismo, se considera que la

geopolítica es una ciencia política dinámica, que se encarga de estudiar el desenvolvimiento político, histórico y económico de un Estado con respecto a sus proyecciones a futuro, teniendo en cuenta la influencia de factores de tipo histórico, raciales, geográficos y económicos para extraer conclusiones de carácter político que benefician al Estado. (Gutiérrez, 2015, p. 5)

Otro concepto que se debe tener en cuenta es el que propone uno de los autores más representativos del tema, Sir Halford J. Mackinder (1861-1947):

La geopolítica estudia los hechos políticos considerando al mundo como una unidad cerrada, en la que tienen repercusión según la importancia de los Estados. En este sentido, los factores geográficos, principalmente, la situación, extensión, población, recursos y comunicaciones de los Estados, si bien no son determinantes, tienen gran importancia y deben ser tenidos en cuenta para orientar la política exterior. (Rosales, 2005, p. 28)

Además, por ejemplo, Leiva (2014) toma como referencia la opinión que tiene Augusto Pinochet Ugarte sobre esta ciencia: 
La geopolítica es una rama de las ciencias políticas que, basada en los conocimientos geográficos, históricos, sociológicos, económicos, estratégicos y políticos, pasados y presentes, estudia en conjunto la vida y desarrollo de una masa humana organizada en un espacio terrestre, analizando sus múltiples y recíprocas influencias (sangre suelo) para deducir sus objetivos y estudiar sus proyecciones, con el fin de lograr en el futuro un mayor bienestar y felicidad en el pueblo. (p. 1)

Este criterio forma parte de la diversidad de concepciones sobre el tema, las cuales aportan diferentes puntos de vista que enriquecen el análisis de la realidad. En este sentido, es pertinente traer a colación el siguiente comentario: "Hablar de geopolítica en estos tiempos, es referirse a un concepto que ha sido demonizado, según mi parecer, por su concepción eminentemente ideológica, totalitaria y militarista (Lacoste, 2006; citado por Dantec, 2007, p. 72). Para otros autores, la geopolítica clásica está "vinculada a un saber instrumental y 'enmascarador' de los intereses del Estado y las prácticas hegemónicas" (Tuathail, 1998; citado por Preciado, 2010, p. 65).

Como se aprecia, sería complejo formular un concepto único de un concepto tan dinámico como la geopolítica, pues este dinamismo se debe precisamente al carácter cambiante de los grupos humanos asentados en un territorio: las sociedades son fuerzas vivas que cambian y se ajustan a condiciones ambientales, económicas, políticas y territoriales. Este escenario muestra con claridad que la geopolítica analiza la interacción entre los intereses de dichos actores y la forma como pueden obtener el poder, y es desde esta perspectiva que se puede analizar la existencia de principios éticos que determinen si sus actuaciones se rigen por elementos más allá de sus propios fines.

De esta forma, se evidencia que en el análisis geopolítico también es importante cuestionar la forma como interactúan o se desarrollan los intereses de los países. Por esta vía se hace posible pensar la ética en el escenario geopolítico, por cuanto existen bienes e intereses comunes que determinan esta forma de actuar. Este es el caso de la seguridad global, la cual se desarrolló después de los atentados del 9-11, cuando todo el planeta comprendió que podía ser atacado en cualquier momento y lugar, lo cual muestra con claridad la coexistencia de intereses nacionales y comunes. En consecuencia, este tipo de criterios permite evidenciar el desarrollo desde el Estado de una ética que 
determine su forma de actuar dentro del sistema internacional, partiendo de su mayor muestra de poder, como lo son las Fuerzas Militares.

\section{Perspectiva colombiana de la ética militar}

A partir de los elementos descritos, la presente sección profundiza en la forma como esta dimensión ética militar se presenta en el Estado colombiano. Particularmente, para revisar el desarrollo institucional de la ética dentro de las Fuerzas Militares se debe iniciar con un análisis no solo de los diferentes instrumentos que constituyen la propia doctrina de la Fuerza, sino también de la perspectiva que tiene el Estado sobre dicha ética militar. Por ello, se comienza estudiando los postulados constitucionales del país, dado que es el instrumento que se encarga de determinar la forma de conducción de la nación.

En este sentido, el artículo 222 constitucional dice que "la ley determinará los sistemas de promoción profesional, cultural y social de los miembros de la Fuerza Pública. En las etapas de su formación, se les impartirá la enseñanza de los fundamentos de la democracia y de los Derechos Humanos" (énfasis de los autores). Este artículo se debe estudiar por unidad de materia con el artículo 217 constitucional con el fin de comprobar cuál es el sustento ético constitucional de Colombia para las Fuerzas Militares. Dicho artículo menciona que "las Fuerzas Militares tendrán como finalidad primordial la defensa de la soberania, la independencia, la integridad del territorio nacional y del orden constitucional' (énfasis de los autores). Con estos dos componentes normativos se evidencia que la norma constitucional determina los fines legítimos que deben alcanzar las Fuerzas Militares, al tiempo que determina la forma como deben ser alcanzados con los medios adecuados. De este modo queda claro que la Constitución exige que a los miembros de las Fuerzas Militares se les imparta "la enseñanza de los fundamentos de la democracia y de los Derechos Humanos" (CN, art 222), lo cual denota la forma de conducta que la Carta Magna espera de los miembros de las Fuerzas Militares, en otras palabras, los imperativos éticos que deben seguir.

Como se ha dicho, el fundamento constitucional del régimen especial castrense se encuentra en el artículo 217, el cual permite que las Fuerzas 
Militares tengan un régimen disciplinario propio. Por esta razón, el legislador expidió en el año 2003 el régimen disciplinario para los miembros de las Fuerzas Militares (Ley 836 de 2003), en el cual se señala que las faltas disciplinarias tipificadas difieren abiertamente de las que contempla la Ley 734 de 2002 en función de la misión particular que cumplen las Fuerzas Militares. Sin embargo, no solo se encuentran bastas diferencias en la parte sustancial, sino que también el aspecto procesal presenta varias divergencias. Ahora bien, aunque la jurisprudencia ha reiterado que la descripción de las faltas en que pueden incurrir los militares y las sanciones que se les pueden imponer son diferentes a las de cualquier otro organismo estatal, toda vez que las faltas guardan estrecha relación con la especial función militar, el procedimiento que se sigue para aplicar tales sanciones sí puede ser igual al contemplado en la Ley 734 de 2002 (Sentencia C-432). Esto evidencia que en esta materia se está ante la presencia de un tipo específico del derecho disciplinario, dado que su condición particular y exclusiva va dirigida a regular el actuar público de funcionarios del Estado en condiciones y situaciones especiales, muy diferentes a las que se presentan en los demás servidores públicos, en razón de su superior función constitucional, de su organización, jerarquía, formación y misión como miembros de las Fuerzas Militares. Esta normativa se ratifica en

la jurisprudencia constitucional, la cual ha reconocido una libertad de configuración legislativa, al disponer que le corresponde al legislador establecer autónoma y libremente las reglas del debido proceso administrativo, siempre que no exista una restricción de tipo constitucional, derivada de sus principios, valores, garantías y derechos (presunción de inocencia, garantía de no ser juzgado dos veces por el mismo hecho, principio de favorabilidad cuando se deban imponer sanciones, etc.), que limite el ámbito de ejercicio de su competencia (Gómez, 2012, p. 122)

En complemento con la Constitución Política de Colombia, también se debe tener en cuenta el Plan Nacional de Desarrollo (PND) vigente de la actual presidencia, denominado "Pacto por Colombia, Pacto por la equidad", dado que los dos documentos determinan los objetivos políticos o fines estratégicos que deben alcanzar las Fuerzas Militares y, en ese sentido, la perspectiva ética de los miembros de las Fuerzas Militares que espera el Estado. En el PND, 
específicamente en el pacto "Seguridad, autoridad y orden para la libertad", se determinan las conductas que se esperan de las Fuerzas Militares así como los principios que deben regir la ética militar, como "fortalecer las capacidades de defensa y seguridad nacional [...] y el marco institucional y las capacidades estratégicas de defensa y seguridad nacional para proteger la soberanía, los intereses y los activos estratégicos de la Nación”. Asimismo, se establece que se fortalecerá “el Consejo de Seguridad Nacional”, se adaptará “una Estrategia de Seguridad Nacional para la protección de los intereses nacionales" y se "implementará e institucionalizará, a partir de un enfoque de acción conjunta, coordinada e interinstitucional, un mecanismo que integre las capacidades de las Fuerzas Militares, para enfrentar los riesgos que afectan la defensa nacional".

Dentro de estos postulados se pueden identificar con claridad objetivos estratégicos que definen claramente los principios éticos que deben seguir las Fuerzas Militares, como el objetivo estratégico encaminado a fortalecer "los programas de educación para continuar con la profesionalización del personal militar y policial”. Es decir, queda claro que la formación de los miembros de las Fuerzas Militares ${ }^{5}$, entre ellas la ética, es de gran importancia para el Estado porque de esta manera pueden mejorar su capacidad.

En este sentido y de acuerdo con el PND, uno de los principales requerimientos a las Fuerzas Militares es salvaguardar los intereses nacionales, de forma que cada una de sus actuaciones debe estar encaminada a cumplir este objetivo. No obstante, se reitera la importancia de que los medios con que puedan alcanzar dichos intereses sean legítimos, para lo cual el PND define la filosofía o forma que debe regir a cada autoridad.

Dentro de estos principios se puede destacar el "Pacto de cero tolerancia a la corrupción y a la falta de transparencia”, con el cual el Estado busca implementar "líneas de acción dirigidas a romper con los paradigmas culturales que valoran más la cultura del atajo y la sensibilización respecto a la corresponsa-

5 Estos programas comprenden el mejoramiento del sistema educativo en términos de metodología, currículos e infraestructura para el desarrollo de los procesos formativos, la acreditación de alta calidad institucional y de los programas de las escuelas de formación, así como el fortalecimiento de los procesos de internacionalización y bilingüismo. Lo anterior se realizará de la mano con acciones que tiendan a fortalecer la rigurosidad de los procesos de selección, méritos y reconocimientos para la prestación del servicio militar, de tal forma que se estimule la incorporación de los mejores jóvenes. 
bilidad en la corrupción”, con lo cual queda claro cuáles son los imperativos éticos que el Estado espera de las Fuerzas Militares.

Por su parte, el Plan Estratégico Institucional (PEI) del Ejército Nacional — publicado en su página institucional— presenta al comienzo como elementos base las palabras y políticas de comando, y luego se divide en cinco capítulos: "Direccionamiento estratégico", "Contexto estratégico", "Marco conceptual. Guía de planeamiento estratégico 2016 -2018”, “Transformación del Ejército Nacional" y "Planeación estratégica institucional".

Respecto a las palabras y las políticas de comando, el comandante del Ejército Nacional para su época, el señor General Ricardo Gómez Nieto, orientó la labor de la institución en la responsabilidad constitucional que tiene el soldado de atender las necesidades de la nación, para lo cual es necesario desarrollar procesos de transformación que permitan afrontar los nuevos desafíos y lograr la modernización y adaptación de la institución a un entorno cambiante para apoyar la estabilización del país. Esos procesos han tenido muchos retos y dificultades, los cuales se han podido superar gracias a la convicción y la renovación del pensamiento del soldado colombiano, pero sobre todo a la convicción de que la paz es el camino efectivo para un país con mayores oportunidades. Por eso, "el Ejército Nacional, comprometido integralmente con la arquitectura de una paz estable y duradera, continúa trabajando en esa ruta bajo una dinámica reflexiva, inteligente y transparente" (Ejército Nacional de Colombia, 2016). En este sentido, es una oportunidad que tiene la institución para cumplir su misión de garantizar la defensa y la seguridad nacionales, así como para contribuir de esta manera al desarrollo integral en el ámbito socio-económico y en los demás factores que permiten el pleno desarrollo del país.

En el primer capítulo, "Direccionamiento estratégico", se describen los principios y valores del Ejército Nacional, los cuales constituyen la base fundamental del soldado colombiano y se consolidan en una cultura del ser, el pensar y el actuar como servidores íntegros al servicio de toda una comunidad. $\mathrm{Al}$ respecto, se debe tener en cuenta que "la sociedad y sus instituciones fundamentales no podrán subsistir ni progresar a menos que todos sus miembros profesen y vivan unos mismos principios y valores éticos basados en la ley 
natural, que tiene su fundamento en el Creador" (Contreras, 2002, p. 14). Estos principios y valores, a su vez, están alineados con la misión y la visión de la institución, que se deriva del artículo 217 de la Constitución Política de Colombia. Específicamente, el propósito de la misión es "conducir operaciones militares orientadas a defender la soberanía, la independencia y la integridad territorial, con el fin de proteger la población civil y sus recursos, para lograr un país en paz y estable, contribuyendo a la seguridad y al desarrollo social y económico", lo cual demuestra el compromiso incondicional que tiene el Ejército con la ciudadanía y los lineamientos del gobierno nacional.

En este mismo capítulo del Plan Estratégico Institucional del Ejército Nacional se establecen cuatro líneas estratégicas, las cuales hacen referencia a las estrategias contempladas en el Plan de Campaña Victoria Plus ${ }^{6}$ y que tienen los siguientes aspectos: (1) garantizar la soberanía y la seguridad nacional para mantener la integridad del territorio de cualquier amenaza interna o externa; (2) contribuir a la transición del proceso de paz, estabilizando y consolidando el territorio nacional y, por último pero no menos importante, (3) transformar la institución con el propósito de tener unos hombres y mujeres más profesionales que puedan enfrentar los desafíos de las nuevas amenazas que afronta la nación. Estas líneas estratégicas orientan el esfuerzo del Ejército Nacional para respaldar las decisiones del gobierno nacional y de los gobiernos regionales, con lo cual se logra una mayor interacción entre organizaciones (Ejércitoentidades gubernamentales) y se mejora la satisfacción de las necesidades de los ciudadanos.

De igual manera, en este capítulo se identifica la base filosófica, la estructura organizacional y, por último, el fortalecimiento de la transparencia y la acción integral, elementos fundamentales que le permiten al Ejército Nacional establecer el soporte estructural para formular los objetivos estratégicos dentro de la legitimidad e integridad institucional.

6 El Plan Estratégico Victoria Plus sirve de orientación a todos los planes y estrategias militares desarrollados para garantizar la tranquilidad de los colombianos con el objetivo de, en resumen, potenciar los esfuerzos conjuntos, coordinados, interagenciales e interinstitucionales de las Fuerzas Militares, la Policía Nacional y la Fiscalía General de la Nación para neutralizar la amenaza persistente del ELN, el GaO-residual, los Gao Pelusos, Puntilleros y Clan del Golfo. 
En el segundo capítulo, "Contexto estratégico", se desarrolla el ambiente operacional que vive el país, el cual se define como volátil, incierto, complejo y ambiguo (VICA), factores que demandan un liderazgo asertivo y una planificación estratégica eficiente del Ejército Nacional. Según Velázquez et al. (2019),

los procesos de paz, la pobreza, la desigualdad, el narcotráfico, la presencia de nuevos actores ilegales y la inestabilidad regional, entre otras variables, afectan la realidad nacional, por ende, también afectan las condiciones que rodean las operaciones del Ejército y lo obligan a romper paradigmas, cambiar e innovar en el empleo del poder militar.

En concordancia, a través del Plan Estratégico Institucional el Ejército Nacional busca responder efectivamente a estos tiempos de incertidumbre, con el fin de afrontar y gestionar de manera efectiva cambios rápidos y adaptarse a estos contextos. Las amenazas que afectan la nación han evolucionado, debido a que Colombia es una región compleja donde convergen diversas corrientes políticas y sociales, además de la gran diversidad étnica y cultural que caracteriza cada departamento.

En las últimas décadas estas regiones han tenido que enfrentar un alto grado de polarización política y social, de modo que se han visto afectadas por la crisis económica global y nacional. Pese a que Colombia está en una zona rica en recursos naturales, su desarrollo socioeconómico es limitado y, por lo tanto, la desigualdad social sigue presente en casi todo el país. En virtud de esta situación, se requiere un Ejército preparado y más profesional, capaz de responder no solo a las realidades nacionales, sino también a los requerimientos específicos de cada una de las regiones y los territorios.

Por su parte, el tercer capítulo, "Marco conceptual. Guía de planeamiento estratégico 2016-2018”, describe el proceso de alineación estratégica, con el cual se pretende transversalizar cada una de las estrategias de la institución con los documentos rectores del sector y de política, vistos como instrumentos esenciales para el planeamiento y la proyección de la Fuerza: el artículo 217 de la Constitución Política, el Plan Nacional de Desarrollo 2014-2018 del gobierno nacional, la Política de Defensa y Seguridad, el Plan Estratégico del Sector Defensa, el Plan Estratégico Militar de las Fuerzas Militares 2030, 
el Plan de Transformación, los Planes de Guerra y de Campaña "Espada de Honor" y "Victoria" del Comando General de las Fuerzas Militares y del Ejército Nacional, entre otros. Documentos que son analizados para identificar los lineamientos que le competen al Ejército Nacional y que se integran a los objetivos del Plan Estratégico Institucional de la institución (figura 1).

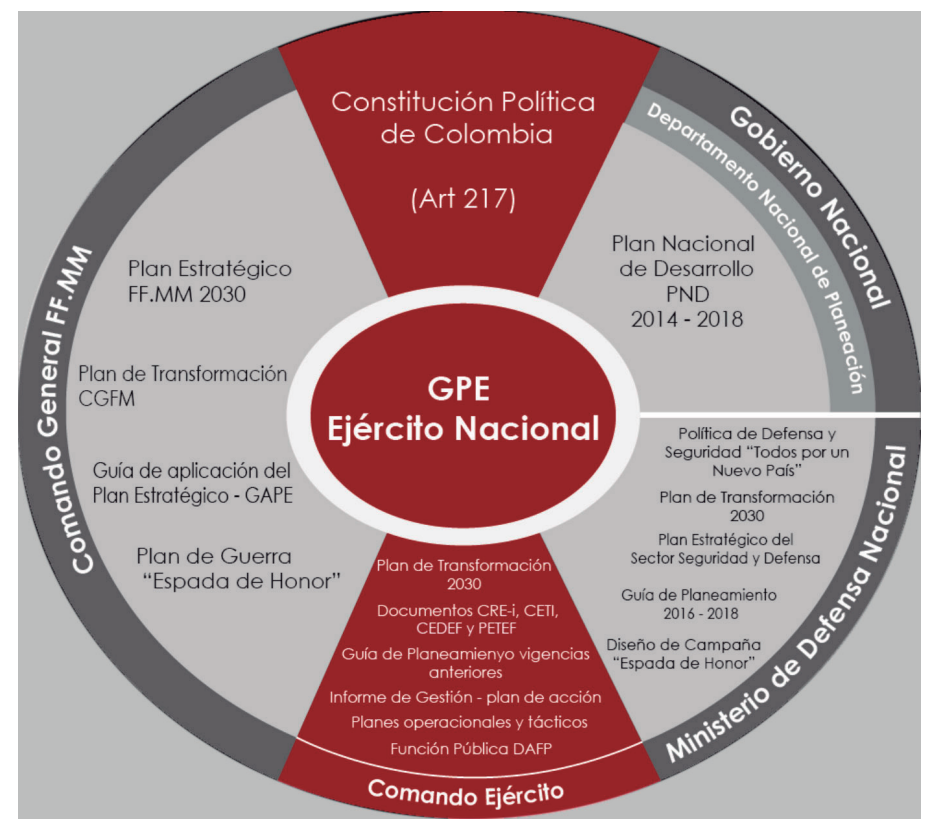

Figura 1. Documentos rectores del Planeamiento Estratégico Institucional.

Fuente: Departamento de Planeación CEDE 5, Plan Estratégico Institucional (2016-2018).

Este proceso le permite al Ejército Nacional relacionar y agrupar cada una de las estrategias establecidas en los documentos rectores para, luego de un diagnóstico, estructurar y formular los objetivos estratégicos del Plan Estratégico Institucional. Por consiguiente, este documento le sirve al Ejército Nacional para alcanzar los objetivos que se fija para cada vigencia, los cuales son traducidos en planes de acción anuales desde la perspectiva del fortalecimiento e interacción con los Comandos de División, Brigada y Unidad Táctica, de tal manera que se refleje y aplique el planeamiento estratégico de forma integral para cumplir los objetivos propuestos (Ejército Nacional de Colombia, 2016). 
El cuarto capítulo, "Transformación del Ejército Nacional", explica que este proceso le permite a la institución proyectarse a futuro para anticiparse a los retos del mañana, siendo una Fuerza más profesional, preparándose desde el hoy para seguir cumpliendo con la misión institucional: "El Ejército Nacional debe garantizar la calidad, pertinencia y sostenibilidad de sus operaciones en el tiempo, esto, en un escenario en el que los desafíos y las amenazas a la seguridad y la defensa del país son cada día más complejos" (Ejército Nacional de Colombia, 2016, p. 97). Por consiguiente, la transformación demanda un cambio significativo en aspectos de innovación, desempeño, competitividad, formación y cultura organizacional, entre otros, con el fin de lograr una modificación sustancial del diseńo y la estructura de la institución y su naturaleza doctrinal.

En este sentido, el Ejército Nacional debe generar una actitud permanente de renovación, innovación y aprendizaje, en concordancia con las características de la situación del entorno de las nuevas amenazas internas y externas, lo cual demanda una transformación dinámica individual y colectiva de la organización. De acuerdo con Calderón et al. (2009),

un factor de éxito de las organizaciones, en el contexto global que se vive actualmente en el mundo, es su capacidad para cambiar de manera apropiada, trascendiendo la idea de adaptación para llegar al de transformación, esto es, desarrollar la capacidad para modificar no solo estructuras, sino también para replantearse elementos más profundos, como valores, comportamiento, cultura y métodos de operación, manteniendo una actitud de renovación y aprendizaje permanente. (p. 2)

De esta manera, el proceso de transformación es de suma importancia para el Ejército Nacional, toda vez que le permite afrontar los retos de los diferentes ámbitos a los cuales se enfrenta, no solo en lo que tiene que ver con la defensa y la seguridad, sino también en la interacción con las comunidades más vulnerables y en los temas sociales, económicos, ambientales, entre otros. Además, esta transformación requiere del trabajo interagencial e integral en conjunto con las autoridades regionales, siempre en beneficio del desarrollo multidimensional de la población civil.

Finalmente, el quinto capítulo, "Planeación estratégica institucional", establece cómo se desarrolla el proceso de planeación estratégica en la Fuerza, el 
cual está basado en los artículos 26 y 29 de la Ley 152 de 1994, en relación con las prioridades que el gobierno determina en el Plan Nacional de Desarrollo, así como en el marco fiscal y normativo en lo concerniente a elaborar un Plan Indicativo Cuatrienal con los planes de acción anuales, instrumentos que se constituyen en la base para la evaluación de los resultados.

Este proceso de planeación permite la retroalimentación en cada una de sus cinco fases o al finalizar el proceso, lo cual facilita que sea flexible frente a los cambios del entorno o a los factores de inestabilidad. Asimismo, este proceso cuenta con la participación de personal idóneo y experto de cada una de las áreas de planeación, así como de los diferentes miembros de los equipos directivos de la Fuerza, lo cual permite realizar un diagnóstico y formular estrategias concisas y aterrizadas a la situación actual que vive el país y la institución (figura 2).

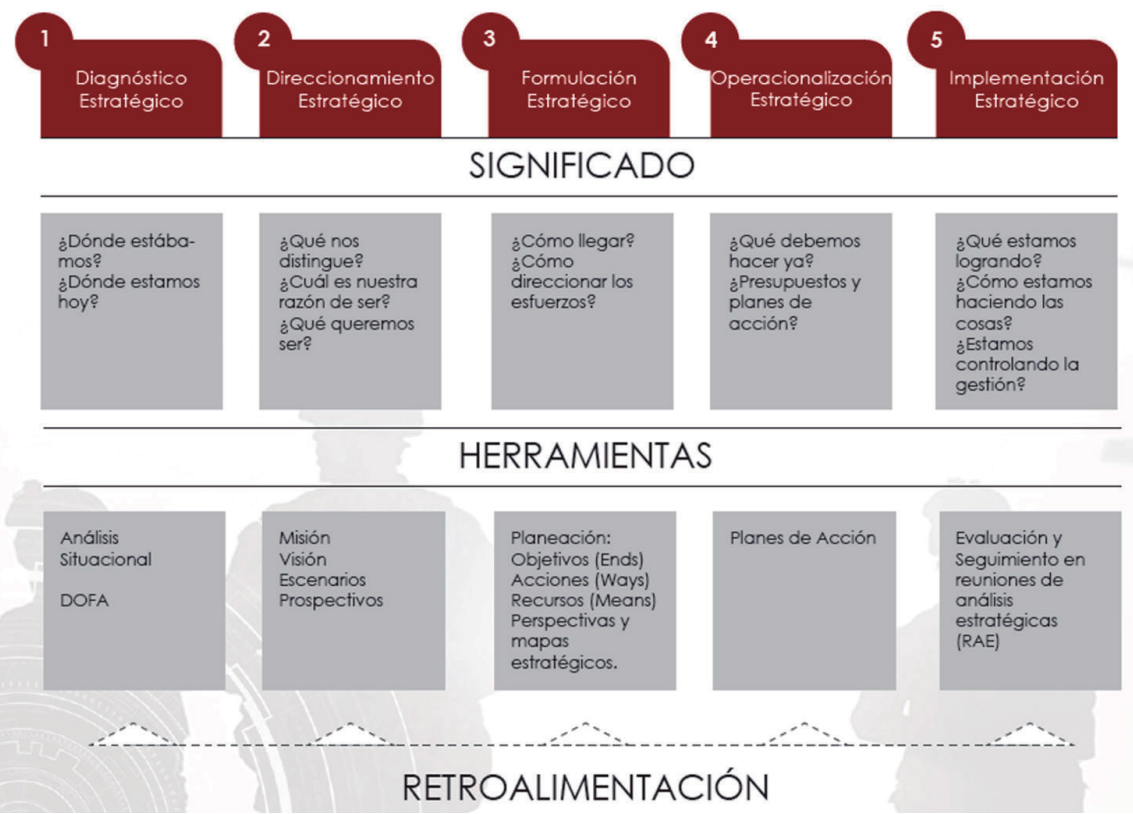

Figura 2. Proceso de Planeación Estratégica.

Fuente: DiPLE - CEDE 5, tomado de Plan Estratégico Institucional 2016-2018. 


\section{Conclusiones}

Como resulta evidente, la reflexión sobre la consolidación y proyección geopolítica de un Estado tiene un papel muy importante en el control y la administración de la violencia en el territorio, lo cual hace que las Fuerzas Armadas sean un actor fundamental en este tipo de procesos.

Estas acciones, que se relacionan con la estrategia y la racionalidad de los Estados, cuentan con unos contornos claros de carácter ético que reconocen su naturaleza democrática. Asimismo, estos valores proyectados socialmente enmarcan las acciones de los militares y se construyen a través de los documentos institucionales, desde los principios constitucionales hasta la implementación de las estrategias.

Dicho elemento axiológico, que llega hasta la decisión de los individuos vinculados con las organizaciones militares, es un factor de análisis que debe acompañar también los ejercicios propios de la geopolítica, tanto desde la perspectiva analítica como en la delimitación de las cuestiones estratégicas y los medios para alcanzar los objetivos del Estado en la escala global, estatal y local.

\section{Referencias}

Aragón, F. (2010). Colombia y su poder geopolítico [Trabajo de pregrado, Pontificia Universidad Javeriana]. Repositorio Universidad Javeriana. https://repository.javeriana. edu.co/bitstream/handle/10554/7704/tesis369.pdf;jsessionid=CB46517CAC79EA051F0E77AE2E0B7114? sequence $=1$

Ballesteros, M. Aznar, F. Ruiz F., Laborie, M. Bolaños J., \& Díez J. (2014). Panorama geopolitico de los conflictos. Instituto de Estudios Estratégicos del Gobierno Español.

Baños, P. (2016). Asi se domina el mundo. Ariel.

Calderón Hernández, G., Cuartas Castaño, J., \& Álvarez Giraldo, C. M. (2009). Transformación organizacional y prácticas innovadoras de gestión humana. Innovar, 19(35), 151-166. https://revistas.unal.edu.co/index.php/innovar/article/view/28773

Dantec, F. (2007). Politica y estrategia. Academia Nacional de Estudios Políticos y Estratégicos Chile.

Departamento de Planeación CEDE5. (2016). Plan Estratégico Institucional 2016-2018. https://www.ejercito.mil.co/s_i_g/planeacion_estrategica_347095

Ejército Nacional de Colombia (2016). PEI Plan Estratégico Institucional.

Grajales Becerra, P. A. (2010, junio 17). Geopolítica. https://www.slideshare.net/paulinita77/ geopolitica-4529495 
Gutiérrez, J. (2015). Geopolítica, recursos naturales y zonas estratégicas en Colombia [Trabajo de pregrado, Universidad Militar Nueva Granada]. Repositorio UMNG. https:// repository.unimilitar.edu.co/bitstream/handle/10654/13560/GEOPOLITICA,\%20 RECURSOS\%20NATURALES\%20Y\%20ZONAS\%20ESTRATEGICAS\%20EN\%20 COLOMBIA\%20.pdf;jsessionid=782C48E010256A07B4BE51C36AF0CFD6?sequence $=2$

Leiva, J. (2014). Geopolitica y nuevo orden mundial. Instituto de Investigación Jurídica UNAH.

Ley 836. (2003). Por la cual se expide el reglamento del régimen disciplinario para las Fuerzas Militares. Congreso de la República. https://www.funcionpublica.gov.co/eva/gestornormativo/norma.php?i=8818

Moliner, J. (2018a). ¿Por qué es importante la ética militar? Revista Española de Defensa.

Moliner, J. (2018b). ¿Qué es la ética militar? http://www.ieee.es/Galerias/fichero/docs_ marco/2018/DIEEEM16-2018_EticaMilitar_JAMoliner.pdf

Moliner, J. (2020). La ética militar y su importancia para el militar profesional [Tesis de doctorado, Escuela Internacional de Doctorado EIDUNED]. Repositorio institucional._http:// e-spacio.uned.es/fez/view/tesisuned:ED-Pg-SegInt-Jamoliner

Núñez, G. F., \& Ferro, Ó. A. C. (2017). Geopolítica contemporánea y análisis de factores relevantes a escala global. Razón Crítica, (3), 111-144.

Preciado, J. (2010). La construcción de una geopolítica crítica desde América Latina y el Caribe. Hacia una agenda de investigación regional. Revista de Estudios sobre Espacio y Poder, 1(1), 65-94. https://revistas.ucm.es/index.php/GEOP/article/view/ GEOP1010120065A/13427

Rosales, G. (2005). Geopolitica y geoestrategia. Liderazgo y poder. Universidad Militar Nueva Granada.

Sentencia C-432. (2004). Corte Constitucional, magistrado ponente Rodrigo Escobar Gil. https://www.corteconstitucional.gov.co/relatoria/2004/C-432-04.htm

Velásquez, N., Colin, M., \& Hernández, O. (2019). Gestión y finanzas para gerentes de proyecto. Universidad Externado de Colombia.

Wead, S. (2015). La ética, el combate y la decisión de matar de un soldado. Military Review, (septiembre-octubre), 3-17. 